\title{
ANALYSIS OF THE THERMOPHYSICAL PROPERTIES AND INFLUENCING FACTORS OF VARIOUS ROCK TYPES FROM THE GUIZHOU PROVINCE
}

\author{
Song Xiaoqing ${ }^{1,2}$,Jiang Ming ${ }^{2, *}$,and Xiong Peiwen ${ }^{1}$ \\ ${ }^{1}$ Geo-engineering investigation institute of guizhou province, 550008 Guiyang ,China \\ ${ }^{2} 111$ Geological Party,Guizhou Bureau of Geology and Mineral Exploration \& Development, 550008 Guiyang,China
}

\begin{abstract}
A series of analyses have been carried out on a number of rock types from the Guizhou Province to investigate their thermophysical properties. A total of 433 samples from 14 types of rock were collected, tested and analyzed. It was found that in this province, the average thermal conductivity of the samples ranged between $1.516 \pm 0.264$ and $5.066 \pm 0.521 \mathrm{~W} /(\mathrm{m} \cdot \mathrm{K})$, the average specific heat capacity varied from $0.272 \pm 0.042$ to $0.603 \pm 0.096 \mathrm{~kJ} /\left(\mathrm{kg} \cdot{ }^{\circ} \mathrm{C}\right)$, and the average thermal diffusion coefficients were from $0.752 \pm 0.331$ to $2.854 \pm 0.368 \mathrm{MJ} /\left(\mathrm{m}^{3} \cdot \mathrm{K}\right)$. The older rocks always had higher thermal conductivity and thermal diffusion. Thermal conductivity and thermal diffusion of rocks are positively correlated with the mineral content of high thermal conductivity species, but the situation for the specific heat capacity is the opposite. With increasing mineral particle size, the thermal conductivity and thermal diffusion coefficient also increase, but the relationship with specific heat capacity is not obvious. The thermal conductivity and thermal diffusion coefficient of rocks increases under water saturated conditions compared to dry conditions, but the specific heat capacity decreases.
\end{abstract}

\section{Introduction}

The thermophysical properties of rocks mainly refer to their thermal behavior. The most important of these include thermal conductivity, thermal diffusion, specific heat capacity, and radioactive heat generation rate[1]. The thermal properties of rocks directly affect the generation, storage and transfer of heat within the Earth, and are indispensable parameters for research into temperature distribution on the Earth's surface and its interior[2]. For practical applications, an example of the importance of these properties is in the design of ground source heat pump systems, where the heat transfer between the system's heat exchanger, tunnel systems, and the surrounding geological bodymust be determined[3,4], as well as being relevant to the engineering of tunnels, subways, etc [5,6].

During the past 30 years, research has been carried out on the thermophysical properties of rocks at the regional scale of northwest China, north China and southeast China within the context of gas, oil, and geothermal energyexploration[7-12]. However, no research in relation to the Guizhou Province or even southwest China in general has been carried out to the authors' knowledge. To remedy this, a field campaign was undertaken in Guizhou Province to collect samples from different lithologies and eras in order to carry out measurements of the thermal conductivity, thermal diffusivity and specific heat capacity of the region's rocks. In total, 433 samples, covering the main rock types and geological units of the region, were collected and analyzed. The resulting measurements therefore provide basic data for research into the province's geothermal potential, hydrocarbon accumulation and geotechnical engineering.

\section{Geological overview of the study area}

Guizhou Province is located in southwest China and is part of the Qiangtang-Yangtze-South China landmass. The strata inside the region are well developed, with outcrops from the Neoproterozoic to the Quaternary. It is in fact one of the regions in China having the most completely developed sedimentary stratum. Guizhou strata from the different eras show a strong regularity in terms of their spatial distribution (Figure 1). A large area of Neoproterozoic erathem is located around Guizhou Liping, Congjiang, and Rongjiang, in the southeast of the province, as well as in the Fanjingshan area in the northeast. Among the above regions, it is mainly metamorphic rock stratum, with lower Paleozoic strata mainly distributed in the eastern, northern, and central parts of Guizhou. Among the above regions, Cambrian strata are the most widely distributed, with dolomite being the main lithology, while Upper Paleozoic strata 
are mainly distributed in the southern and northwestern parts of the province. Limestone is the main rock type, consisting almost entirely of biological limestone and biological crumbs limestone, with some reef limestone and dolomite distributed in Devonian system high field and Carboniferous pendulum group. Strata from the Triassic are mainly distributed in the southwest, central and northern parts of province. Except for their upper parts, which is formed by limestone, these units are mostly dolomite. The dolomites of all strata have a tendency for increasing grain size and stratum thickening with decreasing depth. Jurassic and Cretaceous units are mainly distributed in the Chishui and Xishui areas, which are located in the northern part of Guizhou. Both the Jurassic system and Chishui-Xishui Cretaceous belt are mainly comprised of continental red rock series, while the Cretaceous systems in other parts of the province are composed of conglomerate and red rock series[13].

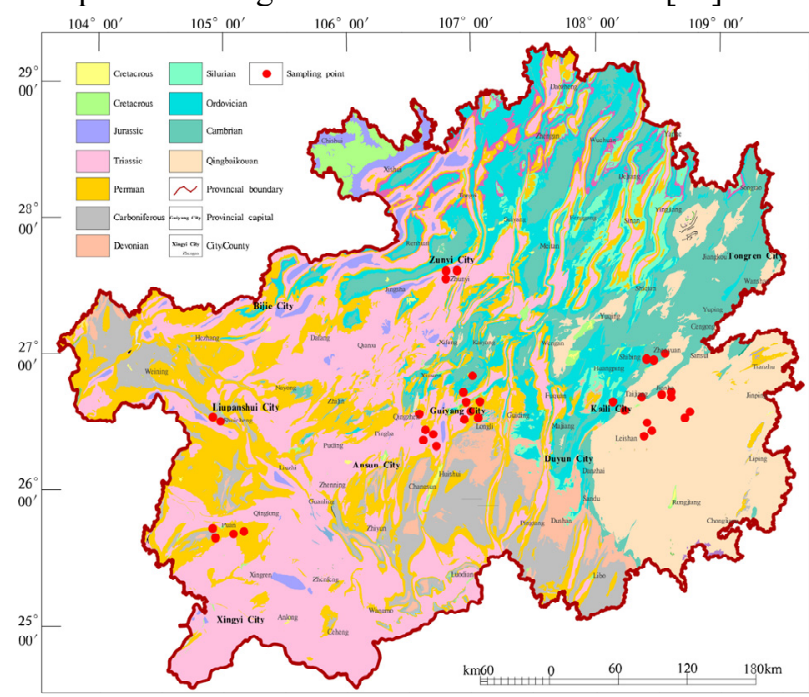

Fig.1. Guizhou rock stratum distribution and sampling site layout

\section{Sampling and Testing}

A total of 433 samples from 18 groups are collected, covering 9 categories from the Cretaceous (E) to the Qingbaikou System (Pt). These samples essentially cover all of the main types of rock outcrop in Guizhou Province, including limestone, marl, dolomite, argillaceous dolomite, quartz sandstone, shale, mudstone, siltstone, carbonaceous shale, conglomerate, basalt, tuff, slate and blastopsammitic rock. The first seven of these include rocks of multiple stratigraphic ages, and the latter seven are from units of single stratigraphic ages. In order to ensure the representativeness and reliability of the test data, more than 6 pieces of rocks from the same stratum were sampled for testing. Sampling points are distributed between Guiyang city, Zunyi city, Liupanshui city, Pu'an county, Kaili city and Zhenyuan county (Figure 1), covering all geographical areas of the province.

The thermophysical parameters measured include the thermal conductivity, thermal diffusivity, and specific heat capacity. All rocks were tested under dry and wet conditions, except for the mudstone and shale, which were tested only in a dry state. The rock samples were processed by the key laboratory dealing with renewable energy of the Ministry of Education in Shandong Jianzhu University, and measured following indoor thermophysical property tests. A Hot Disk (Model: 2500S) thermal constant analyzer was adopted to carry out the measurements, based on the principle of Transient Plane Source Method (TPS). The measurement ranges of the instrument are: 0.01 to $400 \mathrm{~W} /(\mathrm{m} \cdot \mathrm{K})$ for thermal conductivity, 0.1 to $100 \mathrm{~mm}^{2} / \mathrm{S}$ for heat diffusivity, and $\leq$ $5 \mathrm{MJ} /\left(\mathrm{m}^{3} \cdot \mathrm{K}\right)$ for specific heat capacity, with all having an accuracy of $< \pm 2 \%$. This instrument and the employed method had previously been successfully applied to rock thermophysical property parameter testing[14-16]. When measuring the rocks, a probe is sandwiched between two samples to act as the heat source. Over a set time period, the sample's temperature was recorded. The resistance of nickel, which changes with temperature, is taken to indicate the temperature changes. The thermophysical parameters of the tested samples can then be obtained through analysis of the measurements and calculations.

\section{Results Analysis}

\subsection{Thermal Conductivity}

The thermal conductivity (units $\mathrm{W} /(\mathrm{m} \cdot \mathrm{K})$ ) of rock represents the heat passing through a unit area within a unit time when the temperature difference between two points in the direction the heat is flowing differs by $1^{\circ} \mathrm{C}[2]$. The thermal conductivity values measured from the Guizhou rocks (dry) are mostly between 2.0 and $5.0 \mathrm{~W} /(\mathrm{m} \cdot \mathrm{K})$ (Table 1), with average values between $1.516 \pm 0.264$ and $5.066 \pm 0.521 \mathrm{~W} /(\mathrm{m} \cdot \mathrm{K})$. The maximum value measured was $5.828 \mathrm{~W} /(\mathrm{m} \cdot \mathrm{K})$, and the minimum $1.033 \mathrm{~W} /(\mathrm{m} \cdot \mathrm{K})$.

The thermal conductivity of a rock is a function of its mineral composition, structure, porosity, water content, temperature and pressure. In the same type of rock, the thermal conductivity may vary in different samples due to varying proportions of the mineral components, the different structures, and porosities. Regardless of the stratigraphic factors, the rock samples tested in this paper are classified into 14 types of thermal conductivity (dry) according to the rock types and are listed in Table 1. These are, from highest to lowest: dolomite $4.54 \pm 0.823$ $\mathrm{W} /(\mathrm{m} \cdot \mathrm{K})$, quartz sandstone $3.932 \pm 0.805 \mathrm{~W} /(\mathrm{m} \cdot \mathrm{K})$, conglomerate $\quad 3.438 \pm 0.809 \quad \mathrm{~W} /(\mathrm{m} \cdot \mathrm{K}), \quad$ siltstone $3.362 \pm 0.536 \mathrm{~W} /(\mathrm{m} \cdot \mathrm{K})$, tuff $3.355 \pm 0.378 \mathrm{~W} /(\mathrm{m} \cdot \mathrm{K})$, argillaceous dolomite $3.182 \pm 1.213 \mathrm{~W} /(\mathrm{m} \cdot \mathrm{K})$, slate $3.04 \pm 0.488 \mathrm{~W} /(\mathrm{m} \cdot \mathrm{K})$, blastopsammitic rock $2.909 \pm 0.433$ $\mathrm{W} /(\mathrm{m} \cdot \mathrm{K})$, limestone $2.868 \pm 0.256 \mathrm{~W} /(\mathrm{m} \bullet \mathrm{K})$, marl rock $2.699 \pm 0.259 \mathrm{~W} /(\mathrm{m} \bullet \mathrm{K})$, shale $2.021 \pm 0.575 \mathrm{~W} /(\mathrm{m} \bullet \mathrm{K})$, $\begin{array}{lll}\text { carbonaceous shale } 2.213 \pm 0.0 .202 & 2.213 \pm 0.0 .202\end{array}$ $\mathrm{W} /(\mathrm{m} \cdot \mathrm{K})$, basalt $2.084 \pm 0.055 \mathrm{~W} /(\mathrm{m} \cdot \mathrm{K})$, mudstone $1.806 \pm 0.326 \mathrm{~W} /(\mathrm{m} \cdot \mathrm{K})$. The thermal conductivity of dolomite, quartz sandstone, conglomerate and argillaceous dolomite can be seen to vary greatly. This is mainly because these rocks belong to different stratigraphic ages and display structural differences and various mineral compositions. The thermal conductivity 
of the other rocks, however, vary little, with standard deviations between 0.055 and $0.575 \mathrm{~W} /(\mathrm{m} \bullet \mathrm{K})$.

By comparing the thermal conductivity values of the most widely distributed dolomite, limestone, mudstone and siltstone in Guizhou with similar rock types in the north[17], northwest[18], and southeast[10] parts of China (Table 2), it is found that the thermal conductivity of dolomite, limestone and mudstone from
Guizhou is very close to that of rocks from the north of China, with differences of less than $10 \%$. However, they show quite large differences with those from northwest and southeast China, where the Guizhou dolomites show thermal conductivities higher than those from the northwest and southeast, while the Guizhou mudstone thermal conductivity values are lower than those from the northwest and southeast.

Table 1. Rock thermal conductivity statistics table in Guizhou area

\begin{tabular}{|c|c|c|c|c|c|c|c|}
\hline \multirow{2}{*}{\multicolumn{2}{|c|}{ Stratigraphy }} & \multirow[b]{2}{*}{ Lithology } & \multirow[b]{2}{*}{$\begin{array}{l}\text { Number } \\
\text { (Piece) }\end{array}$} & \multicolumn{2}{|c|}{ Dry sample } & \multicolumn{2}{|c|}{ Saturated sample } \\
\hline & & & & $\begin{array}{c}\text { Range } \\
{[\mathrm{W} /(\mathrm{m} \cdot \mathrm{K})]}\end{array}$ & $\begin{array}{c}\text { Avgrage } \pm \text { Standard } \\
\text { deviation } \\
{[\mathrm{W} /(\mathrm{m} \cdot \mathrm{K})]}\end{array}$ & $\begin{array}{c}\text { Range } \\
{[\mathrm{W} /(\mathrm{m} \cdot \mathrm{K})]}\end{array}$ & $\begin{array}{c}\text { Avgrage } \pm \text { Standard } \\
\text { deviation } \\
{[\mathrm{W} /(\mathrm{m} \cdot \mathrm{K})]}\end{array}$ \\
\hline \multirow{2}{*}{\multicolumn{2}{|c|}{ Cretacrous }} & Mudstone & 16 & $1.142 \sim 2.100$ & $1.795 \pm 0.263$ & - & - \\
\hline & & Gravel & 29 & $2.152 \sim 5.581$ & $3.438 \pm 0.810$ & $2.742 \sim 5.354$ & $3.593 \pm 0.646$ \\
\hline \multirow{2}{*}{\multicolumn{2}{|c|}{ Jurassic }} & Siltstone & 30 & $2.424 \sim 4.733$ & $3.362 \pm 0.536$ & $2.401 \sim 4.870$ & $3.900 \pm 0.671$ \\
\hline & & Quartz sandstone & 10 & $2.467 \sim 4.796$ & $3.602 \pm 0.736$ & $2.375 \sim 4.925$ & $4.123 \pm 0.668$ \\
\hline \multirow{7}{*}{ Triassic } & \multirow[b]{2}{*}{ Huaxi Fm } & Dolomite & 15 & $2.750 \sim 4.732$ & $3.553 \pm 0.621$ & $2.860 \sim 4.514$ & $3.517 \pm 0.629$ \\
\hline & & $\begin{array}{c}\text { Argillaceous } \\
\text { dolomite }\end{array}$ & 15 & $1.394 \sim 2.934$ & $2.151 \pm 0.389$ & $2.310 \sim 3.666$ & $2.924 \pm 0.335$ \\
\hline & \multirow[b]{2}{*}{ Anshun Fm } & Dolomite & 15 & $3.156 \sim 5.026$ & $4.473 \pm 0.499$ & $2.338 \sim 5.909$ & $4.423 \pm 0.664$ \\
\hline & & $\begin{array}{c}\text { Argillaceous } \\
\text { dolomite }\end{array}$ & 13 & $3.365 \sim 5.446$ & $4.373 \pm 0.590$ & $4.156 \sim 6.044$ & $4.985 \pm 0.627$ \\
\hline & Yelang Fm & Mudstone & 15 & $1.900 \sim 2.265$ & $2.106 \pm 0.096$ & - & - \\
\hline & \multirow{2}{*}{ Daye Fm } & Limestone & 15 & $2.737 \sim 2.782$ & $2.650 \pm 0.105$ & $2.550 \sim 2.854$ & $2.730 \pm 0.079$ \\
\hline & & Marlstone & 10 & $2.162 \sim 2.779$ & $2.562 \pm 0.153$ & $2.341 \sim 2.802$ & $2.581 \pm 0.142$ \\
\hline \multirow{3}{*}{ Permian } & $\begin{array}{c}\text { Changxing } \\
\text { Fm }\end{array}$ & Mudstone & 15 & $1.119 \sim 2.189$ & $1.516 \pm 0.264$ & - & - \\
\hline & Emeishan Fm & Basalt & 30 & $1.984 \sim 2.202$ & $2.084 \pm 0.055$ & $1.686 \sim 2.271$ & $2.077 \pm 0.100$ \\
\hline & Maokou Fm & Limestone & 15 & $2.815 \sim 3.579$ & $3.085 \pm 0.161$ & $2.961 \sim 3.990$ & $3.168 \pm 0.258$ \\
\hline \multirow{3}{*}{\multicolumn{2}{|c|}{ Carboniferous Datang Fm }} & Quartz sandstone & 10 & $2.850 \sim 5.410$ & $3.736 \pm 0.731$ & $3.912 \sim 5.901$ & $4.554 \pm 0.526$ \\
\hline & & Marlstone & 7 & $2.420 \sim 2.870$ & $2.547 \pm 0.139$ & $2.052 \sim 2.573$ & $2.451 \pm 0.167$ \\
\hline & & Shal & 15 & $1.033 \sim 2.763$ & $1.729 \pm 0.482$ & - & - \\
\hline \multicolumn{2}{|c|}{ Devonian Gaozhaitian Fm } & Quartz sandstone & 10 & $2.984 \sim 5.270$ & $4.458 \pm 0.666$ & $3.916 \sim 6.294$ & $5.017 \pm 0.713$ \\
\hline \multicolumn{2}{|c|}{ Silurian Gaozhaitian Fm } & Marlstone & 11 & $2.542 \sim 3.439$ & $2.920 \pm 0.238$ & $2.707 \sim 3.413$ & $2.985 \pm 0.179$ \\
\hline \multirow[t]{3}{*}{ Cambrian } & $\begin{array}{c}\text { Loushanguang } \\
\text { Fm }\end{array}$ & Dolomite & 30 & $3.582 \sim 5.828$ & $5.066 \pm 0.521$ & $3.257 \sim 6.091$ & $5.210 \pm 0.669$ \\
\hline & Mingxinsi Fm & Shal & 11 & $1.323 \sim 2.769$ & $2.419 \pm 0.437$ & - & - \\
\hline & Niutitang Fm & Carbonaceous shale & 6 & $1.779 \sim 2.367$ & $2.123 \pm 0.202$ & $1.877 \sim 2.412$ & $2.236 \pm 0.818$ \\
\hline \multirow{3}{*}{\multicolumn{2}{|c|}{ Qingbaikouan }} & Tuff & 30 & $2.599 \sim 4.258$ & $3.355 \pm 0.378$ & $2.003 \sim 4.390$ & $3.303 \pm 0.494$ \\
\hline & & Slate & 30 & $1.847 \sim 4.061$ & $3.041 \pm 0.488$ & $2.127 \sim 4.162$ & $3.171 \pm 0.442$ \\
\hline & & Metamorphic-sand & 30 & $2.121 \sim 3.952$ & $2.910 \pm 0.378$ & $2.047 \sim 3.903$ & $3.030 \pm 0.419$ \\
\hline
\end{tabular}

Table 2. Comparison of rock thermal conductivity statistics in other regions

\begin{tabular}{|c|c|c|c|c|c|c|}
\hline \multirow{2}{*}{ Lithology } & \multicolumn{2}{|c|}{$\begin{array}{c}\text { North China } \\
\text { (Chen } \text { et al.,1998) }\end{array}$} & \multicolumn{2}{c|}{$\begin{array}{c}\text { Northwest China } \\
\text { (Wang } \text { et al.,1995) }\end{array}$} & \multicolumn{2}{c|}{$\begin{array}{c}\text { Southeast } \\
\text { (Xiong } \text { et al.,1994) }\end{array}$} \\
\cline { 2 - 7 } & $\begin{array}{c}\text { Thermal } \\
\text { conductivity } \\
{[\mathrm{W} /(\mathrm{m} \cdot \mathrm{K})]}\end{array}$ & $\begin{array}{c}\text { Number } \\
\text { (Piece) }\end{array}$ & $\begin{array}{c}\text { Thermal } \\
\text { conductivity } \\
{[\mathrm{W} /(\mathrm{m} \cdot \mathrm{K})]}\end{array}$ & $\begin{array}{c}\text { Number } \\
\text { (Piece })\end{array}$ & $\begin{array}{c}\text { Thermal } \\
\text { conductivity } \\
{[\mathrm{W} /(\mathrm{m} \cdot \mathrm{K})]}\end{array}$ & $\begin{array}{c}\text { Number } \\
\text { (Piece) }\end{array}$ \\
\hline Mudstone & $1.97 \pm 0.16$ & 3 & $2.506 \pm 0.688$ & 26 & $3.59 \pm 0.1 .19$ & 49 \\
\hline Siltstone & $2.51 \pm 0.71$ & 17 & $1.811 \pm 0.578$ & 21 & - & - \\
\hline Dolomite & $4.34 \pm 1.33$ & 45 & $3.501 \pm 0.922$ & 4 & $3.30 \pm 0.67$ & 8 \\
\hline Limestone & $2.86 \pm 1.13$ & 21 & $3.192 \pm 0.724$ & 28 & $3.33 \pm 0.56$ & 56 \\
\hline
\end{tabular}



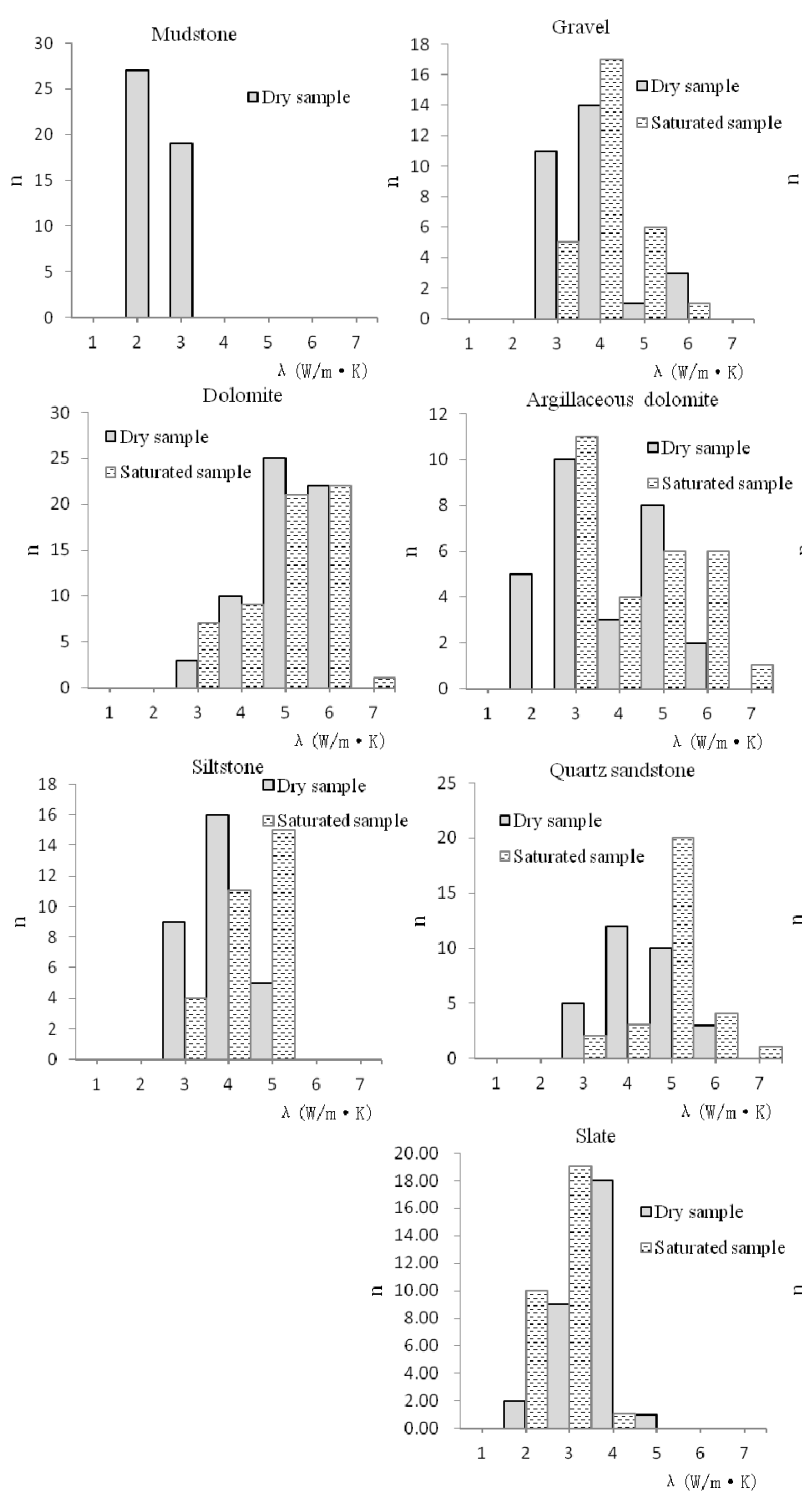

Fig.2. Histograms of Rock Thermatc

\subsection{Specific Heat Capacity}

Rock specific heat capacity refers to the heat absorbed by a unit mass of rock for a $1{ }^{\circ} \mathrm{C}$ temperature rise (or the heat released by a $1{ }^{\circ} \mathrm{C}$ temperature decrease) in $\mathrm{kJ} /\left(\mathrm{kg} \cdot{ }^{\circ} \mathrm{C}\right)$. The specific heat capacity (dry) of the samples from Guizhou range between 0.174 and $0.873 \mathrm{~kJ} /\left(\mathrm{kg} \cdot{ }^{\circ} \mathrm{C}\right)$ (Table 3), for which, the measured average value is between $0.272 \pm 0.042$ and $0.603 \pm 0.096 \mathrm{~kJ} /\left(\mathrm{kg} \cdot{ }^{\circ} \mathrm{C}\right)$, with a maximum value of $0.873 \mathrm{~kJ} /\left(\mathrm{kg} \cdot{ }^{\circ} \mathrm{C}\right)$ and a minimum of $0.174 \mathrm{~kJ} /\left(\mathrm{kg} \cdot{ }^{\circ} \mathrm{C}\right)$. The average specific heat capacity
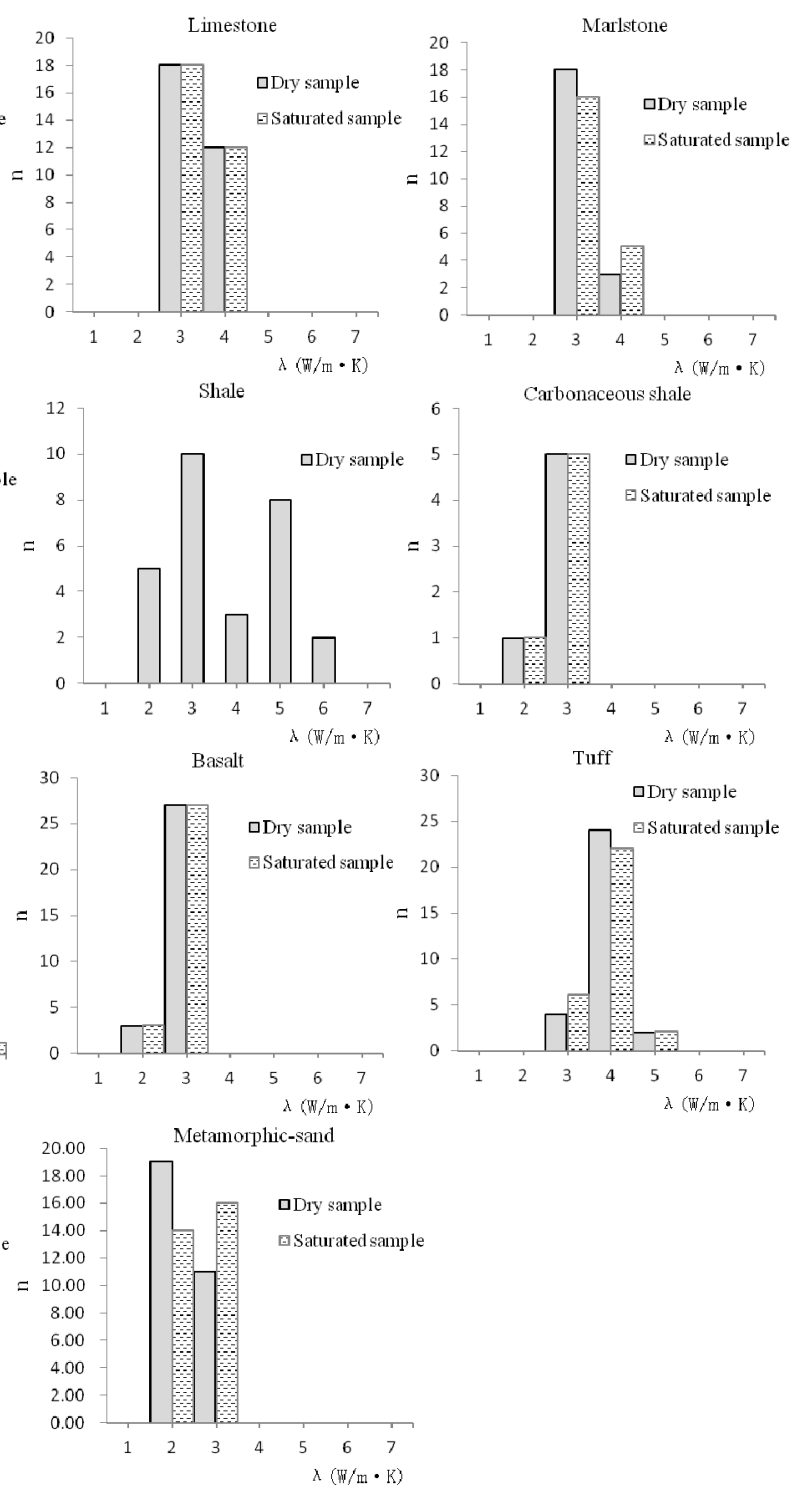

1 Conductivities in Guizhou Area

values from high to low are respectively: basalt $0.603 \pm 0.096 \mathrm{~kJ} /\left(\mathrm{kg} \cdot{ }^{\circ} \mathrm{C}\right)$, conglomerate $0.566 \pm 0.192$ $\mathrm{kJ} /\left(\mathrm{kg} \cdot{ }^{\circ} \mathrm{C}\right)$, dolomite $0.553 \pm 0.082 \mathrm{~kJ} /\left(\mathrm{kg} \cdot{ }^{\circ} \mathrm{C}\right)$, marl $0.545 \pm 0.057 \mathrm{~kJ} /\left(\mathrm{kg} \cdot{ }^{\circ} \mathrm{C}\right)$, tuff $0.528 \pm 0.085 \mathrm{~kJ} /\left(\mathrm{kg} \cdot{ }^{\circ} \mathrm{C}\right)$, limestone $0.515 \pm 0.079 \mathrm{~kJ} /\left(\mathrm{kg} \cdot{ }^{\circ} \mathrm{C}\right)$, blastopsammitic rock $0.502 \pm 0.069 \mathrm{~kJ} /\left(\mathrm{kg} \cdot{ }^{\circ} \mathrm{C}\right), \quad$ mudstone $0.493 \pm 0.126$ $\mathrm{kJ} /\left(\mathrm{kg} \cdot{ }^{\circ} \mathrm{C}\right)$, slate $0.487 \pm 0.099 \mathrm{~kJ} /\left(\mathrm{kg} \cdot{ }^{\circ} \mathrm{C}\right)$, argillaceous dolomite $0.454 \pm 0.106 \mathrm{~kJ} /\left(\mathrm{kg} \cdot{ }^{\circ} \mathrm{C}\right)$, shale $0.411 \pm 0.087$ $\mathrm{kJ} /\left(\mathrm{kg} \bullet{ }^{\circ} \mathrm{C}\right)$, carbonaceous shale $0.388 \pm 0.077 \mathrm{~kJ} /\left(\mathrm{kg} \cdot{ }^{\circ} \mathrm{C}\right)$, quartz sandstone $0.368 \pm 0.069 \mathrm{~kJ} /\left(\mathrm{kg} \cdot{ }^{\circ} \mathrm{C}\right)$, and siltstone $0.272 \pm 0.042 \mathrm{~kJ} /\left(\mathrm{kg} \cdot{ }^{\circ} \mathrm{C}\right)$.

Table 3. Statistics of rock specific heat capacity in Guizhou area

\begin{tabular}{|c|c|c|c|c|c|c|}
\hline \multirow[b]{2}{*}{ Stratigraphy } & \multirow[b]{2}{*}{ Lithology } & \multirow[b]{2}{*}{$\begin{array}{l}\text { Number } \\
\text { (Piece) }\end{array}$} & \multicolumn{2}{|c|}{ Dry sample } & \multicolumn{2}{|c|}{ Saturated sample } \\
\hline & & & $\begin{array}{c}\text { Range } \\
{\left[\mathrm{kJ} /\left(\mathrm{kg} \cdot{ }^{\circ} \mathrm{C}\right)\right]}\end{array}$ & $\begin{array}{c}\text { Avgrage } \pm \text { Standard } \\
\text { deviation } \\
{\left[\mathrm{kJ} /\left(\mathrm{kg} \cdot{ }^{\circ} \mathrm{C}\right)\right]}\end{array}$ & $\begin{array}{c}\text { Range } \\
{\left[\mathrm{kJ} /\left(\mathrm{kg} \cdot{ }^{\circ} \mathrm{C}\right)\right]}\end{array}$ & $\begin{array}{c}\text { Avgrage } \pm \text { Standard } \\
\text { deviation } \\
{\left[\mathrm{kJ} /\left(\mathrm{kg} \cdot{ }^{\circ} \mathrm{C}\right)\right]}\end{array}$ \\
\hline \multirow{2}{*}{ Cretacrous } & Mudstone & 16 & $0.174 \sim 0.567$ & $0.427 \pm 0.124$ & - & - \\
\hline & Gravel & 29 & $0.293 \sim 0.873$ & $0.566 \pm 0.116$ & $0.200 \sim 0.669$ & $0.470 \pm 0.111$ \\
\hline \multirow{2}{*}{ Jurassic } & Siltstone & 30 & $0.180 \sim 0.376$ & $0.272 \pm 0.042$ & $0.112 \sim 0.446$ & $0.259 \pm 0.081$ \\
\hline & Quartz sandstone & 10 & $0.179 \sim 0.459$ & $0.327 \pm 0.078$ & $0.185 \sim 437$ & $0.276 \pm 0.064$ \\
\hline
\end{tabular}




\begin{tabular}{|c|c|c|c|c|c|c|c|}
\hline \multirow{7}{*}{ Triassic } & \multirow[b]{2}{*}{ Huaxi Fm } & Dolomite & 15 & $0.401 \sim 0.637$ & $0.553 \pm 0.055$ & $0.324 \sim 0.654$ & $0.523 \pm 0.078$ \\
\hline & & $\begin{array}{l}\text { Argillaceous } \\
\text { dolomite }\end{array}$ & 15 & $0.309 \sim 0.514$ & $0.415 \pm 0.059$ & $0.375 \sim 0.553$ & $0.464 \pm 0.046$ \\
\hline & \multirow[b]{2}{*}{ Anshun Fm } & Dolomite & 15 & $0.491 \sim 0.673$ & $0.589 \pm 0.047$ & $0.286 \sim 0.662$ & $0.492 \pm 0.109$ \\
\hline & & $\begin{array}{l}\text { Argillaceous } \\
\text { dolomite }\end{array}$ & 13 & $0.238 \sim 0.821$ & $0.499 \pm 0.128$ & $0.275 \sim 0.521$ & $0.414 \pm 0.077$ \\
\hline & Yelang Fm & Mudstone & 15 & $0.364 \sim 0.585$ & $0.511 \pm 0.063$ & - & - \\
\hline & \multirow{2}{*}{ Daye Fm } & Limestone & 15 & $0.303 \sim 0.636$ & $0.511 \pm 0.092$ & $0.375 \sim 0.597$ & $0.492 \pm 0.061$ \\
\hline & & Marlstone & 10 & $0.449 \sim 0.705$ & $0.560 \pm 0.067$ & $0.279 \sim 0.525$ & $0.451 \pm 0.082$ \\
\hline \multirow{3}{*}{ Permian } & $\begin{array}{c}\text { Changxing } \\
\text { Fm }\end{array}$ & Mudstone & 15 & $0.256 \sim 0.729$ & $0.549 \pm 0.145$ & - & - \\
\hline & Emeishan Fm & Basalt & 30 & $0.409 \sim 0.68$ & $0.603 \pm 0.096$ & $0.237 \sim 0.698$ & $0.572 \pm 0.114$ \\
\hline & Maokou Fm & Limestone & 15 & $0.309 \sim 0.601$ & $0.518 \pm 0.065$ & $0.320 \sim 0.688$ & $0.501 \pm 0.084$ \\
\hline \multirow{3}{*}{\multicolumn{2}{|c|}{ Carboniferous Datang Fm }} & Quartz sandstone & 10 & $0.361 \sim 0.524$ & $0.413 \pm 0.048$ & $0.219 \sim 0.432$ & $0.323 \pm 0.068$ \\
\hline & & Marlstone & 7 & $0.482 \sim 0.567$ & $0.532 \pm 0.028$ & $0.433 \sim 0.587$ & $0.491 \pm 0.049$ \\
\hline & & Shal & 15 & $0.249 \sim 0.535$ & $0.393 \pm 0.095$ & - & - \\
\hline \multicolumn{2}{|c|}{ Devonian Gaozhaitian Fm } & Quartz sandstone & 10 & $0.285 \sim 0.466$ & $0.370 \pm 0.047$ & $0.136 \sim 0.434$ & $0.299 \pm 0.093$ \\
\hline \multicolumn{2}{|c|}{ Silurian Gaozhaitian Fm } & Marlstone & 11 & $0.401 \sim 0.610$ & $0.551 \pm 0.057$ & $0.403 \sim 0.585$ & $0.506 \pm 0.057$ \\
\hline \multirow{3}{*}{ Cambrian } & $\begin{array}{c}\text { Loushanguang } \\
\text { Fm }\end{array}$ & Dolomite & 30 & $0.322 \sim 0.689$ & $0.535 \pm 0.099$ & $0.278 \sim 0.654$ & $0.459 \pm 0.101$ \\
\hline & Mingxinsi Fm & Shal & 11 & $0.313 \sim 0.533$ & $0.433 \pm 0.068$ & - & - \\
\hline & Niutitang Fm & $\begin{array}{c}\text { Carbonaceous } \\
\text { shale }\end{array}$ & 6 & $0.219 \sim 0.441$ & $0.388 \pm 0.077$ & $0.289 \sim 0.452$ & $0.393 \pm 0.063$ \\
\hline \multirow{3}{*}{\multicolumn{2}{|c|}{ Qingbaikouan }} & Tuff & 30 & $0.321 \sim 0.699$ & $0.557 \pm 0.093$ & $0.329 \sim 0.692$ & $0.528 \pm 0.085$ \\
\hline & & Slate & 30 & $0.232 \sim 0.605$ & $0.487 \pm 0.099$ & $0.308 \sim 0.611$ & $0.472 \pm 0.080$ \\
\hline & & Metamorphic-sand & 30 & $0.287 \sim 0.603$ & $0.502 \pm 0.069$ & $0.298 \sim 0.745$ & $0.478 \pm 0.091$ \\
\hline
\end{tabular}

\subsection{Thermal Diffusion Coefficient}

The thermal diffusion coefficient is the ratio of the thermal conductivity to the product of the specific heat capacity and density. It indicates the ability of a rock to become uniform in temperature during heating or cooling. In rocks with large thermal diffusion coefficients, heat is diffused at a high rate over long transmission distances, with the opposite for those rocks with small thermal diffusion coefficients. The thermal diffusion coefficient and thermal conductivity are similar in physical meaning, and the factors affecting the thermal diffusion coefficient and the direction of change are the same as for thermal conductivity. The thermal diffusion coefficients (dry) of the sampled rocks from Guizhou lie mainly between 1.0 and $3.0 \mathrm{~mm}^{2} / \mathrm{s}$ (Table 4). The measured average values are between $0.752 \pm 0.331$ and $2.854 \pm 0.368 \mathrm{MJ} /\left(\mathrm{m}^{3} \cdot \mathrm{K}\right)$, with a maximum value of $4.23 \mathrm{~mm}^{2} / \mathrm{s}$ and a minimum of $0.418 \mathrm{~mm}^{2} / \mathrm{s}$. The thermal diffusion coefficients of the dolomite and clastic rocks containing $\mathrm{SiO}_{2}$ were mainly from 2.0 to $3.0 \mathrm{~mm}^{2} / \mathrm{s}$, which is higher than for the other rocks, mainly because of the higher thermal conductivity of dolomite and quartz[19,20]. The thermal diffusion coefficients of the mudstone and shale are mostly less than $1.0 \mathrm{~mm}^{2} / \mathrm{s}$, which makes them the rock types with the lowest thermal diffusion coefficient in the province. The thermal diffusion coefficients of the other rocks are between 1.0 and $2.0 \mathrm{~mm}^{2} / \mathrm{s}$.

Table 4. Rock thermal diffusion statistics table in Guizhou area

\begin{tabular}{|c|c|c|c|c|c|c|c|}
\hline \multirow{2}{*}{\multicolumn{2}{|c|}{ Stratigraphy }} & \multirow[b]{2}{*}{ Lithology } & \multirow{2}{*}{$\begin{array}{l}\text { Number } \\
\text { (Piece) }\end{array}$} & \multicolumn{2}{|c|}{ Dry sample } & \multicolumn{2}{|c|}{ Saturated sample } \\
\hline & & & & $\begin{array}{l}\text { Range } \\
\left(\mathrm{mm}^{2} / \mathrm{s}\right)\end{array}$ & $\begin{array}{l}\text { Avgrage } \pm \text { Standard } \\
\text { deviation }\left(\mathrm{mm}^{2} / \mathrm{s}\right)\end{array}$ & $\begin{array}{l}\text { Range } \\
\left(\mathrm{mm}^{2} / \mathrm{s}\right)\end{array}$ & $\begin{array}{l}\text { Avgrage } \pm \text { Standard } \\
\text { deviation }\left(\mathrm{mm}^{2} / \mathrm{s}\right)\end{array}$ \\
\hline \multirow{2}{*}{\multicolumn{2}{|c|}{ Cretacrous }} & Mudstone & 16 & $0.629 \sim 2.418$ & $1.086 \pm 0.494$ & - & - \\
\hline & & Gravel & 29 & $1.170 \sim 2.994$ & $1.669 \pm 0.380$ & $1.022 \sim 4.221$ & $2.121 \pm 0.671$ \\
\hline \multirow{2}{*}{\multicolumn{2}{|c|}{ Jurassic }} & Siltstone & 30 & $2.046 \sim 4.230$ & $2.751 \pm 0.481$ & $1.901 \sim 5.939$ & $3.506 \pm 0.915$ \\
\hline & & Quartz sandstone & 10 & $1.560 \sim 3.872$ & $2.674 \pm 0.591$ & $2.649 \sim 4.024$ & $3.600 \pm 0.444$ \\
\hline \multirow{7}{*}{ Triassic } & \multirow[b]{2}{*}{ Huaxi Fm } & Dolomite & 15 & $1.404 \sim 2.465$ & $1.753 \pm 0.323$ & $1.531 \sim 3.794$ & $2.026 \pm 0.587$ \\
\hline & & $\begin{array}{c}\text { Argillaceous } \\
\text { dolomite }\end{array}$ & 15 & $0.799 \sim 1.533$ & $1.223 \pm 0.230$ & $1.377 \sim 2.854$ & $1.660 \pm 0.355$ \\
\hline & \multirow[b]{2}{*}{ Anshun Fm } & Dolomite & 15 & $1.849 \sim 2.494$ & $2.116 \pm 0.182$ & $1.995 \sim 4.141$ & $2.778 \pm 0.714$ \\
\hline & & $\begin{array}{c}\text { Argillaceous } \\
\text { dolomite }\end{array}$ & 13 & $0.999 \sim 3.359$ & $2.214 \pm 0.547$ & $2.016 \sim 4.017$ & $2.973 \pm 0.654$ \\
\hline & Yelang Fm & Mudstone & 15 & $0.799 \sim 1.418$ & $0.963 \pm 0.178$ & - & - \\
\hline & \multirow{2}{*}{ Daye Fm } & Limestone & 15 & $1.150 \sim 2.155$ & $1.434 \pm 0.268$ & $1.205 \sim 2.034$ & $1.508 \pm 0.207$ \\
\hline & & Marlstone & 10 & $1.081 \sim 1.428$ & $1.248 \pm 0.087$ & $1.359 \sim 2.379$ & $1.588 \pm 0.331$ \\
\hline
\end{tabular}




\begin{tabular}{|c|c|c|c|c|c|c|c|}
\hline \multirow{3}{*}{ Permian } & $\begin{array}{c}\text { Changxing } \\
\text { Fm }\end{array}$ & Mudstone & 15 & $0.418 \sim 1.468$ & $0.752 \pm 0.331$ & - & - \\
\hline & Emeishan Fm & Basalt & 30 & $0.876 \sim 1.540$ & $1.024 \pm 0.166$ & $0.828 \sim 2.092$ & $1.102 \pm 0.324$ \\
\hline & Maokou Fm & Limestone & 15 & $1.385 \sim 2.435$ & $1.623 \pm 0.259$ & $1.390 \sim 2.693$ & $1.734 \pm 0.306$ \\
\hline \multirow{3}{*}{\multicolumn{2}{|c|}{ Carboniferous Datang Fm }} & Quartz sandstone & 10 & $1.142 \sim 3.888$ & $2.174 \pm 0.699$ & $2.741 \sim 4.158$ & $3.468 \pm 0.511$ \\
\hline & & Marlstone & 7 & $1.153 \sim 1.386$ & $1.237 \pm 0.089$ & $1.120 \sim 3.392$ & $1.658 \pm 0.717$ \\
\hline & & Shal & 15 & $0.443 \sim 1.860$ & $0.843 \pm 0.383$ & - & - \\
\hline \multicolumn{2}{|c|}{ Devonian Gaozhaitian Fm } & Quartz sandstone & 10 & $2.310 \sim 3.467$ & $2.854 \pm 0.368$ & $2.827 \sim 4.298$ & $3.607 \pm 0.498$ \\
\hline \multicolumn{2}{|c|}{ Silurian Gaozhaitian Fm } & Marlstone & 11 & $1.096 \sim 1.811$ & $1.421 \pm 0.207$ & $1.280 \sim 1.945$ & $1.594 \pm 0.184$ \\
\hline \multirow{3}{*}{ Cambrian } & $\begin{array}{c}\text { Loushanguang } \\
\text { Fm }\end{array}$ & Dolomite & 30 & $1.620 \sim 4.044$ & $2.635 \pm 0.544$ & $2.464 \sim 6.663$ & $3.694 \pm 0.996$ \\
\hline & Mingxinsi Fm & Shal & 11 & $0.425 \sim 1.498$ & $0.997 \pm 0.285$ & - & - \\
\hline & Niutitang Fm & $\begin{array}{c}\text { Carbonaceous } \\
\text { shale }\end{array}$ & 6 & $0.934 \sim 1.465$ & $1.062 \pm 0.184$ & $0.854 \sim 1.464$ & $1.032 \pm 0.202$ \\
\hline \multirow{3}{*}{\multicolumn{2}{|c|}{ Qingbaikouan }} & Tuff & 30 & $1.232 \sim 3.311$ & $1.697 \pm 0.401$ & $1.204 \sim 3.302$ & $1.841 \pm 0.501$ \\
\hline & & Slate & 30 & $1.119 \sim 3.014$ & $1.773 \pm 0.496$ & $1.267 \sim 3.980$ & $1.914 \pm 0.539$ \\
\hline & & Metamorphic-sand & 30 & $1.165 \sim 2.106$ & $1.550 \pm 0.252$ & $1.065 \sim 3.152$ & $1.772 \pm 0.464$ \\
\hline
\end{tabular}

\section{Analysis on Factors Influencing the Rock Thermophysical Property Parameters}

\subsection{Influence of stratum age on a rock's thermophysical parameters}

The values of the thermophysical parameters of the same type of rock differ owing to the different stratum ages. The thermal conductivity of most rocks in the study area shows a specific relationship with age, that is, the older the rock, the higher the thermal conductivity, in agreement with previous work[7,11]. For example, the thermal conductivity of the Jurassic quartz sandstone is $3.602 \pm 0.736 \mathrm{~W} /(\mathrm{m} \bullet \mathrm{K})$, increasing to $3.736 \pm 0.731$ $\mathrm{W} /(\mathrm{m} \cdot \mathrm{K})$ in the Carboniferous stratum, and $4.458 \pm 0.666$ $\mathrm{W} /(\mathrm{m} \cdot \mathrm{K})$ in the Devonian stratum. The thermal conductivity of dolomite in the middle Triassic Huaxi group is $3.553 \pm 0.621 \mathrm{~W} /(\mathrm{m} \cdot \mathrm{K})$, which increases to $4.473 \pm 0.499 \mathrm{~W} /(\mathrm{m} \cdot \mathrm{K})$ in the lower Triassic, and $5.066 \pm 0.521 \mathrm{~W} /(\mathrm{m} \cdot \mathrm{K})$ in the Cambrian. Quartz sandstone and dolomite, limestone, marl and shale in the province display the same trend (Figure 3). Furthermore, as discussed above, since the thermal diffusion coefficient is essentially proportional to the thermal conductivity, it also follows the same tendency, with increasing values with age of the rock.
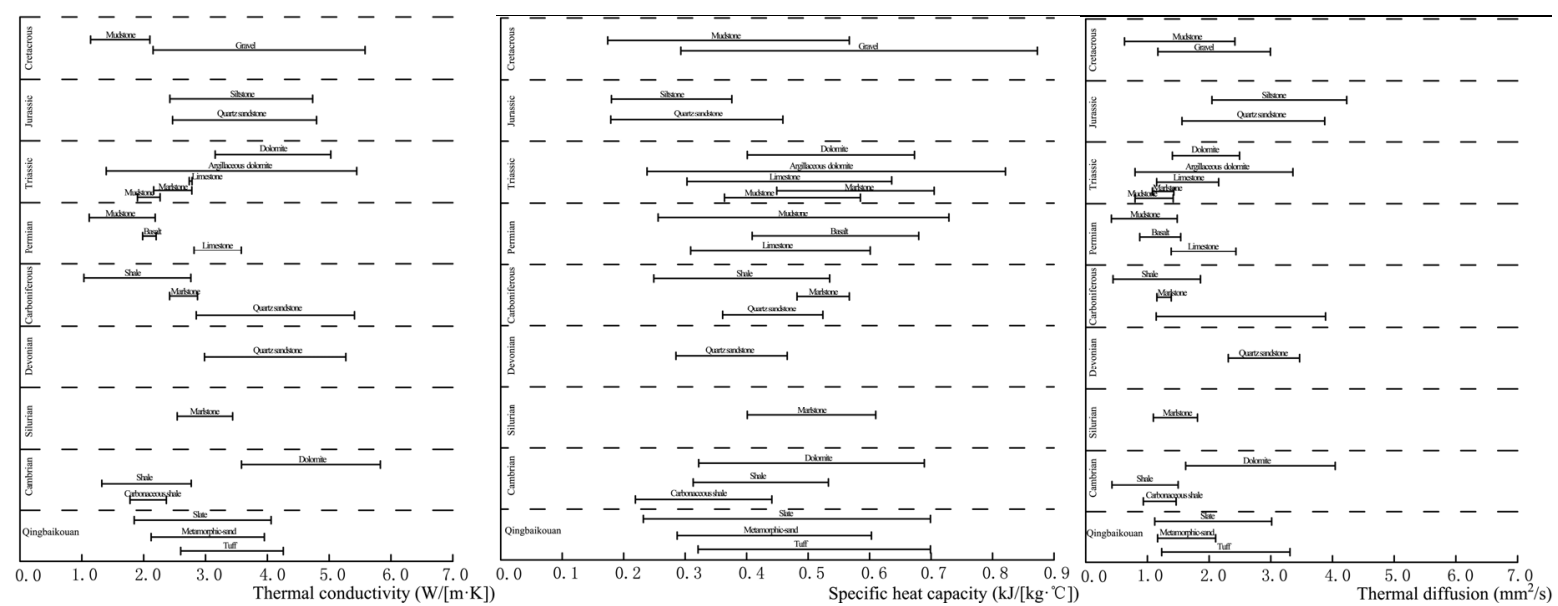

Fig.3. Parameter maps of thermophysical properties of rock in different stratigraphic ages in Guizhou

\subsection{Influence of Mineral Components on Rock Thermophysical Property Parameters}

The thermophysical properties of a rock also vary as a function of its mineral composition. For example, if the mineral content of high thermal conductivity species is high, then the thermal conductivity of the rock will be high. In Guizhou, known as the "sedimentary rock kingdom", quartz, dolomite and calcite are the main mineral components of the rocks. Among them, quartz has the highest thermal conductivity, up to $7.70 \mathrm{~W} /(\mathrm{m} \cdot \mathrm{K})$, while dolomite ranks second with a value of $5.44 \mathrm{~W} /(\mathrm{m} \cdot \mathrm{K})[19,20]$, and calcite the lowest with a thermal conductivity of $3.60 \mathrm{~W} /(\mathrm{m} \cdot \mathrm{K})$. From Table 1 , it can be seen that the thermal conductivity of dolomite and quartz sandstone in the study area is significantly higher than that of limestone, while the thermal conductivity of dolomite is higher than that of quartz sandstone, which may be caused by other factors than mineral content, such as mineral size, and porosity. For rocks with similar mineral compositions, the thermal properties of rocks 
vary greatly due to differences in mineral contents. For example, the higher the $\mathrm{SiO}_{2}$ content in the rock, the greater the thermal conductivity and thermal diffusion coefficient, and the smaller the specific heat capacity. It can be seen from Figure 4 that there is a positive correlation between $\mathrm{SiO}_{2}$ content and thermal conductivity and thermal diffusivity in the blastopsammitic rock, slate, quartz sandstone and siltstone, which have $\mathrm{SiO}_{2}$ as the main mineral component, and a negative correlation with specific heat capacity, consistent with previous work[21].

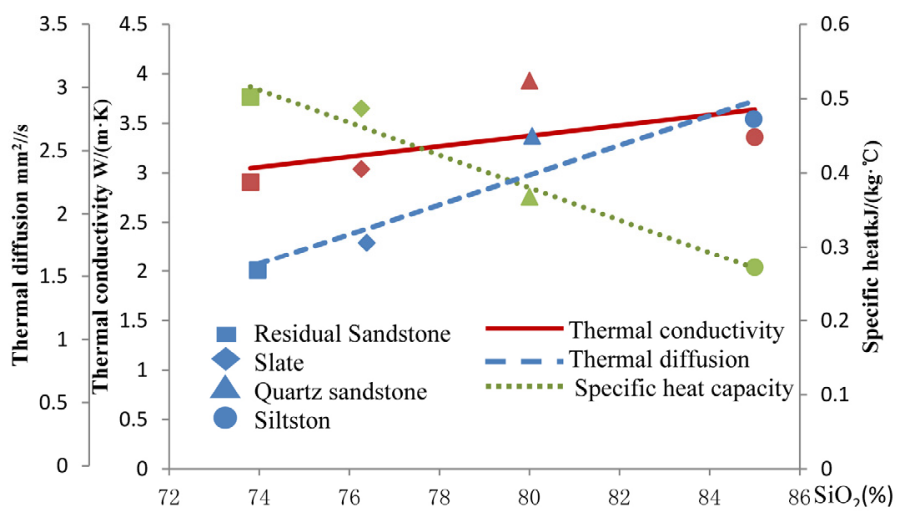

Fig.4. Relationship between different lithologic silica content and thermal properties of rock

\subsection{Influence of mineral particle size on thermophysical properties}

Midttomme, et.al[22]. found that there is a positive correlation between mineral particle size and rock thermal conductivity when studying the thermal properties of artificial rocks. Comparing the size of the mineral particles in the rock with its thermophysical properties, it is found that with increasing mineral particle size, so does the thermal conductivity and thermal diffusion coefficient. For example, considering dolomite mineral particle size, in the Cambrian Loushanguan Group, the particle size is between 0.03 and $0.2 \mathrm{~mm}$, the thermal conductivity of the rock is 5.01 $\mathrm{W} /(\mathrm{m} \cdot \mathrm{K})$, and the thermal diffusion coefficient is 2.502 $\mathrm{mm}^{2} / \mathrm{s}$. In the Triassic Huaxi Group, the particle size of the dolomite minerals is between 0.01 and $0.06 \mathrm{~mm}$, with a thermal conductivity of $4.113 \mathrm{~W} /(\mathrm{m} \cdot \mathrm{K})$ and thermal diffusion coefficient of $1.991 \mathrm{~mm}^{2} / \mathrm{s}$. Considering limestone, in the Permian System Maokou Group, the mineral particle size is between 0.2 and $2.0 \mathrm{~mm}$, with a thermal conductivity of $3.22 \mathrm{~W} /(\mathrm{m} \cdot \mathrm{K})$ and a thermal diffusion coefficient of $1.554 \mathrm{~mm}^{2} / \mathrm{s}$. For the Triassic Daye Group, the particle size of the limestone minerals were between 0.01 and $0.03 \mathrm{~mm}$, with a thermal conductivity of $2.69 \mathrm{~W} /(\mathrm{m} \cdot \mathrm{K})$ and thermal diffusion coefficient of $1.281 \mathrm{~mm}^{2} / \mathrm{s}$. However, the relationship between the specific heat capacity and the mineral particle is not obvious.

\subsection{Influence of water content on the thermophysical properties}

Among the samples, except for those of mudstone and shale, which could not be tested due to looseness after water saturation, all other rocks had their thermophysical properties tested under saturation conditions. It can be seen from Figure 3 that the thermal conductivity measured under different water conditions is different in sandstone, dolomite and limestone. For example, the thermal conductivity of argillaceous dolomite is $3.182 \pm 1.213$. $\mathrm{W} /(\mathrm{m} \cdot \mathrm{K})$ under dry conditions, but increases to $3.94 \pm 1.119 \mathrm{~W} /(\mathrm{m} \cdot \mathrm{K})$ under saturated conditions, an increase of $23.8 \%$. Note, however, that the increase in thermal conductivity of the other samples measured under saturated conditions ranges between $2 \%$ and $17 \%$ higher. This increase may be the result of a heat flow "liquid bridge" forming after water fills the pores, which reduces the thermal resistance between the rock particles[23]. The specific heat capacity of dry rock and saturated rock also differed, but contrary to the thermal conductivity, the specific heat capacity under saturated conditions was reduced by $3.08 \%$ to $21.79 \%$ compared to the values measured under dry conditions. The thermal diffusion coefficient behaved similarly to the thermal conductivity, with an increase of 1 to $16 \%$ when measured under saturated conditions compared to dry.

\section{Conclusions}

Based on the measurements reported above, the following conclusions may be drawn.

(1) The measured values show that the average thermal conductivity of the rock samples collected from the Guizhou Province were between 1.516 \pm 0.264 and $5.066 \pm 0.521 \mathrm{~W} /(\mathrm{m} \cdot \mathrm{K})$. The order of the thermal conductivity (dry) of the various rock types, from highest to lowest, is: dolomite, quartz sandstone, conglomerate, siltstone, tuff, argillaceous dolomite, slate, blastopsammitic rock, limestone, marl, shale, carbonaceous shale, basalt, and mudstone. Among these, the thermal conductivity of the most widely distributed, namely dolomite, limestone and mudstone are very close to that of north China, but quite different from the northwest and southeast. The measured average specific heat capacity (dry) of the samples was between $0.272 \pm 0.042$ and $0.603 \pm 0.096 \mathrm{~kJ} /\left(\mathrm{kg} \cdot{ }^{\circ} \mathrm{C}\right)$. The order of the average values of specific heat capacity, from largest to lowest, are: basalt, conglomerate, dolomite, marl, tuff, 
limestone, blastopsammitic rock, mudstone, slate, argillaceous dolomite, shale, carbonaceous shale, quartz sandstone, and siltstone. The average values of the thermal diffusion coefficient were between $0.752 \pm 0.331$ and $2.854 \pm 0.368 \mathrm{MJ} /(\mathrm{m} 3 \cdot \mathrm{K})$. The thermal diffusion coefficients of the dolomite containing carbonate and clastic rock containing $\mathrm{SiO}_{2}$ mostly ranged between 2.0 and $3.0 \mathrm{~mm}^{2} / \mathrm{s}$, which is higher than other rocks. For example, the thermal diffusion coefficient of mudstone and shale is less than $1.0 \mathrm{~mm}^{2} / \mathrm{s}$, while for the other rocks it was between 1.0 to $2.0 \mathrm{~mm}^{2} / \mathrm{s}$.

(2) The thermophysical properties of rock material are dependent upon their age, mineral composition, structure and particle size. According to the measurements, the thermal conductivity and thermal diffusion coefficient of most rocks from Guizhou Province are related to the age of the stratum, where the older the stratum, the higher the thermal conductivity and thermal diffusion coefficient. Regarding the mineral composition and content, if there is a high content of minerals with high thermal conductivity, then the thermal conductivity of the rock is high, leading in turn to a higher thermal diffusion coefficient of the rock, but a negative correlation with the specific heat capacity. As the size of the mineral particles in the rock increases, the thermal conductivity and thermal diffusion coefficient of the rock also increase. However, the relationship between the specific heat capacity and particle size is not obvious.

(3) Water content has a large influence on the thermophysical properties of the sampled rocks. Under saturated conditions, the thermal conductivity increases by $2 \%$ to $17 \%$ compared with the thermal conductivity under dry conditions, and the thermal diffusion coefficient increases by $1 \%$ to $16 \%$. By contrast, the specific heat capacity is reduced by between $3.08 \%$ and $21.79 \%$.

\section{Acknowledgment}

Foundation item: Guizhou Provincial Bureau of Geology and Mineral Resources Research Project (No.[2015]10),Under the auspices of National Key Research Program of China (No. 2014BAB03B00)

\section{References}

1. $\mathrm{Xu}$ Zhenzhang,1992.A discussion of factors influencing thermophysical characteristics of rocks and their mechanisms. Petroleum exploration and development, 19:85-89.

2. Wang Jiyang,Zhang Jian,Xu Hehua et al.,2015.Geothermics and its Applications.Beijing:Science Press.

3. Yu mingzhi, Fang Zhaohong,2002.A Method for the On-site Testing of Average Thermo-physical Pa-rameters of Underground Rock Soil.Journal of Engineering for Thermal Energy\&Power, 17(5):489-492.

4. Hu Pingfang,Sun Qiming, Yu Zhongyi et al.,2008.Test of Heat Exchang in Ground Heat Exchanger of
Ground Source Heat Punp and Thermal Properties of Soil.Gas\&Heat,28(8):1-4.

5. Li xianglan,Liu shaowen,Xu Ming et al.,2015.Measurement and analysis of thermal porperties of mudstones and shales in the Lower Yangtze,South China.Natural Gas Geoscience,26(8):1525-1533.

6. Kwon S,Kim J S,Lee C S et al.,2011.The thermal and mechanicalproperties rock mass around an underground research tunnel ingranite body[C]// $12^{\text {th }}$ ISRM Congress.International Society for Rock Mechanics.

7. Qiu Nansheng,2003. Haracters of Thermal Conductivity and Radiogenic Heat Production rate in basins of Northwest China.Chinese Journal of Geology,37(2):196-206.

8. Lei Xiaodong,Hu Shengbiao,Li Juan et al.,2018. Thermal properties analysis of bedrock in Beijing. Progress in Geophysics:1-13.

9. Song Ning,Shi Guanghui,Gao Guoqiang et al.,2011.Thermal conductivity of Paleogene-Upper Cretaceous rocks in Subei Basin.Complex Hydrocarbon Reservoirs, 4(1):10-13.

10. Xiong Liangping, $\mathrm{Hu}$ Shenbiao,Wang Jian,1994.Analysis on the thermal conductivity of rocks from se china.Acta Perrologica Sinica,10(3):323-329.

11. Lun Xiwu,Gao Dezhang, Yu Puzhi et al.,2002.Thermal conductivity of the cenozoic layer of east china sea shelf.Oceanologia et Limnolodia Sinica,33(5) :151-159.

12. Yang Shuzhen,Zhang Wenren,Li Guohua et al.,1993.Experimental Research on the Thermal Conductivity of Water-Saturted Rocks and Correction to the Heat Flow Oeserved in Caidam Basin.Acta Perrologica Sinica,9(2):199-204.

13. Dai Chuangu,Wang Xuehua,Chen Jianshu et al.,2017.Regional Geology of China,Guizhou Province.Beijing: Geological Publishing House.

14. Lv Chao,Sun Qiang,Deng Shu et al.,2017.Research on the Thermal Conductivity of Sandstone and Limestone after High Temperature Treatment.Geological Journal of China Universities, 23(4):626-623.

15. Chen Zhenming, $\mathrm{Wu} \mathrm{Xu}, \mathrm{Wang}$ Hongwei et al.,2016.Research of Thermal Conductive Characteris of Granite under High Temperature.Science Technology and Engineering,16(24):193-197.

16. Lü Chao,Sun Qiang,Deng Shu et al.,2017.An experimental study on thermal physical properties of sandstone under high temperature heating.Geology and Exploration,53(4):780-787.

17. Chen Moxiang,Huang Geshan,Xiong Liangping et al.,1988.North China geothermal.Beijing:Science Press.

18. Wang jun,Wang Jian,Shen Jiying et al.,1995.Heat 
Flow in Tarim Basin.Earth Science,20(4):399-404.

19. Birch A F,1948. The effects of Pleistocene climatic variations upon geothermal gradients.American Joernal of Science,53:413-428.

20. Beck A E,1977.Climatically perturbed temperature gradients and their effect on regional and continental heat-flow means.Tectonophysics, 41:17-39.

21. Jorand R,Clauser C,Marquart G,et al,2015. Statistically reliable petrophysical properties of potential reservoir rocks for geothermal energy use and their relation to lithostratigraphy and rock composition:The NE Rhenish Massif and the Lower Rhine Embayment (Germany).Geothermics,53:413-428.

22. Midttomme K,Roaldset E,1998, The effect of grain size on thermal conduc-tivity of quartz sands and silts.Petroleum Geoscience,4:165-172.

23. Li Jishan,2009.Testing and analysis of thermal properties of reservoir rocks.Journal of Daqing Petroleum Institute,33(5):23-26,117-118 